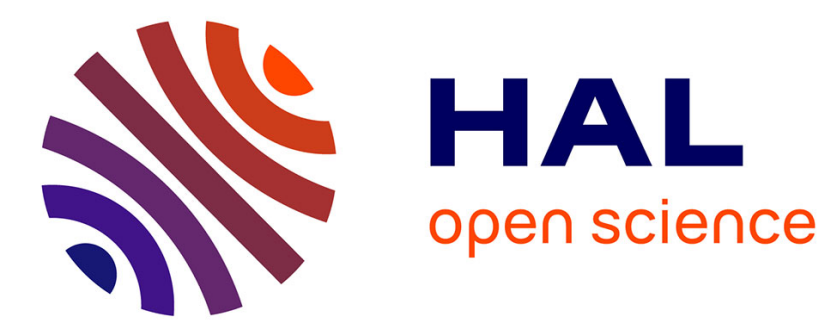

\title{
Compositional Synthesis of Symbolic Controllers for Attractivity Specifications
}

W Alejandro Apaza-Perez, Antoine Girard

\section{To cite this version:}

W Alejandro Apaza-Perez, Antoine Girard. Compositional Synthesis of Symbolic Controllers for Attractivity Specifications. 60th IEEE conference on Decision and Control, Dec 2021, Austin, United States. 10.1109/CDC45484.2021.9682817 . hal-03349984

\section{HAL Id: hal-03349984 \\ https://hal.science/hal-03349984}

Submitted on 21 Sep 2021

HAL is a multi-disciplinary open access archive for the deposit and dissemination of scientific research documents, whether they are published or not. The documents may come from teaching and research institutions in France or abroad, or from public or private research centers.
L'archive ouverte pluridisciplinaire HAL, est destinée au dépôt et à la diffusion de documents scientifiques de niveau recherche, publiés ou non, émanant des établissements d'enseignement et de recherche français ou étrangers, des laboratoires publics ou privés. 


\title{
Compositional Synthesis of Symbolic Controllers for Attractivity Specifications
}

\author{
W. Alejandro Apaza-Perez and Antoine Girard
}

\begin{abstract}
Attractivity specifications consist in driving the state of a system to a target region and to keep it in that region afterwards. In this paper, we develop a compositional approach to symbolic controller synthesis for attractivity specifications. The approach consists in computing iteratively for each subsystem, refinements of the least-violating attractivity controller, and of the associated attractor. The controllers and attractors computed at a given iteration are used at the next iteration as control and external input constraints. The resulting fixed-point algorithm allows us to compute a decentralized attractivity controller for the interconnected system which minimizes the size of the attractor. To illustrate the effectiveness of our approach, we show an application for the temperature regulation of adjacent rooms of a building.
\end{abstract}

\section{INTRODUCTION}

Symbolic control is a computational approach to controller synthesis for nonlinear systems (see e.g. [17], [3] and the references therein). The main concept of symbolic control is that of the symbolic model, also called discrete abstraction, which is a finite state/input approximation of a general continuous dynamical system. When a symbolic model is related to the original system by some formal behavioral relationship such as alternating simulation [17] or feedback refinement [13], controllers designed for the symbolic model can be refined into controllers for the original system. This makes it possible to recourse to automatic controller synthesis techniques for finite state dynamical systems to synthesize controllers for continuous systems (see e.g. [17], [3]). Symbolic control has several advantages. Firstly, it can be applied to very general classes of continuous systems with state and input constraints and subject to bounded disturbances. Secondly, it enables the automatic synthesis of controllers that are "correct-by-design" for various type of specifications such as safety, reachability, attractivity or more complex properties such as those described in Linear Temporal Logic; it also makes it possible to optimize certain performance criteria.

However, the symbolic control approach is subject to the curse of dimensionality and these techniques are effective mostly on low-dimensional dynamical systems. In the past decade, several compositional approaches have been proposed to tackle these scalability issues. The problem of

This work was supported in part by the European Research Council (ERC) under the European Union's Horizon 2020 research and innovation programme (grant agreement No 725144) and by the Agence Nationale de la Recherche (ANR) under Grant HANDY ANR-18-CE40- 0010.

W.A. Apaza-Perez and A. Girard are with the Universite Paris-Saclay, CNRS, CentraleSupélec, Laboratoire des signaux et systèmes, 91190, Gif-sur-Yvette, France \{willy-alejandro.apaza-perez; Antoine.Girard\}al2s.centralesupelec.fr computing a symbolic model for a system by abstracting its components individually has been investigated in [18], [12], [11], [9], [16]. The main challenge though is arguably to design compositional approaches to controller synthesis. With that respect, assume-guarantee contracts offer an appealing theoretical framework [4] where a global system specification is decomposed in contracts specifying the obligations of a component under assumptions on the behavior of other components. Several approaches for compositional synthesis of symbolic controllers make use of such contracts [8], [10], [6], [14]. Some other approaches are based on generalizations of the celebrated small-gain theorem [5], [12], [1], [2]. A third possibility is to start with partial synthesis of controllers on the components, to compute the composition and then to refine the composed controller to enforce the specification at the system level [15].

In this paper, we propose an approach for synthesizing compositionally attractivity controllers. Attractivity specifications consist in driving the state of a system to a target region and to keep it in that region afterwards. Compared to the approaches mentioned above, our approach is iterative and can be interpreted in the assume-guarantee framework as constructing a sequence of assume-guarantee contracts with increasingly strong assumptions and guarantees, captured here by the attractors of the system's components. The proposed approach extends the recent work of [7], which allows to synthesize "least-violating" controllers, to the compositional framework of attractivity controller synthesis. Our approach thus consists in computing iteratively for each subsystem, refinements of the least-violating attractivity controller and of the associated attractor. The controllers and attractors computed at a given iteration are used at the next iteration as control and external input constraints. Then, the resulting fixed point algorithm allows us to compute a decentralized attractivity controller for the interconnected system. The closest work to the approach presented in this paper is [6], where an iterative approach based on leastviolating safety controllers is proposed to design compositionally decentralized safety controllers. In the current work, we address a different type of specifications, namely attractivity. Another difference is that in the current work, we build a sequence of controllers that iteratively improves the behavior of the closed-loop system, while in [6] a controller is only obtained after all iterations have finished.

The structure of this paper is as follows. In Section II, we provide the necessary theoretical background and provide a formal problem formulation. In Section III, we briefly recall the main results of [7] on the synthesis of least-violating 
attractivity controllers. Section IV contains the main contribution of the paper, which is an algorithm to synthesize compositionally a decentralized attractivity controller for an interconnected system. Finally, in Section V, a numerical example of temperature regulation in a building is used to illustrate our approach.

Notation: $\mathbb{R}, \mathbb{R}_{0}^{+}$and $\mathbb{N}$ denote the sets of real, nonnegative real and natural numbers, respectively. For $K \in$ $\mathbb{N} \cup\{+\infty\}$, we define the following sets of integers $\mathbb{N}_{<K}=$ $\{k \in \mathbb{N} \mid k<K\}$ and $\mathbb{N}_{\leq K}=\{k \in \mathbb{N} \mid k \leq K\} . \overline{\mathbb{R}}$ denotes the set of extended real numbers, i.e. $\overline{\mathbb{R}}=[-\infty,+\infty]$. Given two sets $A$ and $B$, a set-valued map $f: A \rightrightarrows B$ is a map from $A$ to the set of subsets of $B$.

\section{Problem Statement}

In this section, we provide a formal statement of the problem under consideration.

\section{A. Transition systems}

We consider a class of transition systems formally defined as follows:

Definition 1: A transition system $\Sigma$ is a quadruple $\Sigma=$ $(X, D, U, F)$ where $X$ is the set of states; $D$ is the set of external inputs; $U$ is the set of control inputs; and $F$ is the transition map $F: X \times D \times U \rightrightarrows X$.

A transition system with no external inputs can be written formally in the form of Definition 1 with a set of external inputs consisting of a unique dummy external input. In that case, with an abuse of notation, we denote $\Sigma=(X, U, F)$.

A transition $x^{\prime} \in F(x, d, u)$ means that $\Sigma$ can evolve from state $x$ to state $x^{\prime}$ under external and control inputs $d$ and $u$. For $x \in X, u \in U$, we denote $F(x, D, u)=\bigcup_{d \in D} F(x, d, u)$. Define the set of enabled control inputs at a state $x \in X$ as

$$
\operatorname{enab}_{F}(x)=\{u \in U \mid \forall d \in D, F(x, d, u) \neq \emptyset\} .
$$

Let us remark that $u$ is enabled at $x$ if and only if there exist successors for all possible values of the external input. If $\operatorname{enab}_{F}(x)=\emptyset$ then $x$ is said to be blocking.

Within the framework of transition systems, we can define (memoryless state-feedback) controllers as follows:

Definition 2: A controller for system $\Sigma$ is a set-valued map $C: X \rightrightarrows U$ such that $C(x) \subseteq \operatorname{enab}_{F}(x)$, for all $x \in X$.

Closed-loop trajectories are then defined as follows:

Definition 3: A sequence $\left(x_{t}\right)_{t=0}^{T}$, where $T \in \mathbb{N} \cup\{+\infty\}$, $x_{t} \in X$, for $t \in \mathbb{N}_{\leq T}$, is called a closed-loop trajectory of system $\Sigma$ with controller $C$ if and only if

$$
\forall t \in \mathbb{N}_{<T}, \exists d_{t} \in D, x_{t+1} \in F\left(x_{t}, d_{t}, C\left(x_{t}\right)\right) .
$$

A trajectory is called maximal if either $T=+\infty$ or $C\left(x_{T}\right)=$ $\emptyset$, it is complete if $T=+\infty$. The set of maximal closedloop trajectories starting from a given initial state $x_{0} \in X$ is denoted by $\mathscr{T}_{\max }\left(\Sigma, C, x_{0}\right)$.

In this paper, we consider uniform attractivity specifications, which are defined as follows:
Definition 4: $X^{*} \subseteq X$ is said to be uniformly attractive from initial state $x_{0} \in X$ for system $\Sigma$ and controller $C$ if there exists $T_{0} \in \mathbb{N}$, such that all maximal trajectories $\left(x_{t}\right)_{t=0}^{T} \in$ $\mathscr{T}_{\max }\left(\Sigma, C, x_{0}\right)$ are complete and satisfy $x_{t} \in X^{*}$, for all $t \geq T_{0}$.

The term uniform refers to the fact that the time bound $T_{0}$ after which the state remains in the target set $X^{*}$ is the same for all trajectories in $\mathscr{T}_{\max }\left(\Sigma, C, x_{0}\right)$. A discussion on the difference between uniform attractivity and (non-uniform) attractivity can be found in [7]. In particular, it should be noted that uniform attractivity cannot be captured using specification formalisms such as linear temporal logic.

\section{B. Interconnected systems}

In this paper, we focus on interconnected transition systems. For the sake of simplicity, we consider systems consisting of two interconnected components but the approach can readily be extended to systems with an arbitrary number of interconnected components.

Hence, let us consider two components given by transition systems $\Sigma_{i}=\left(X_{i}, D_{i}, U_{i}, F_{i}\right), i=1,2$, with $D_{1}=X_{2}$ and $D_{2}=X_{1}$. The interconnected system is then described by a transition system with no external input $\Sigma=(X, U, F)$, where $X=X_{1} \times X_{2}, U=U_{1} \times U_{2}$ and for all $x=\left(x_{1}, x_{2}\right) \in X$, $u=\left(u_{1}, u_{2}\right) \in U$

$$
F(x, u)=F_{1}\left(x_{1}, x_{2}, u_{1}\right) \times F_{2}\left(x_{2}, x_{1}, u_{2}\right) .
$$

Let us consider controllers $C_{i}: X_{i} \rightrightarrows U_{i}$ for components $\Sigma_{i}$, $i=1,2$. We define the associated decentralized controller for $\Sigma, C: X \rightrightarrows U$ given for all $x=\left(x_{1}, x_{2}\right) \in X$ by

$$
C(x)=C_{1}\left(x_{1}\right) \times C_{2}\left(x_{2}\right) .
$$

Let us remark that $C(x) \subseteq \operatorname{enab}_{F_{1}}\left(x_{1}\right) \times \operatorname{enab}_{F_{2}}\left(x_{2}\right) \subseteq$ $\operatorname{enab}_{F}(x)$, so $C$ satisfies Definition 2 .

Let us consider a target set $X^{*}=X_{1}^{*} \times X_{2}^{*}$ where $X_{i}^{*} \subseteq$ $X_{i}, i=1,2$. We now formally define the problem under consideration in this paper:

Problem 5: Synthesize controllers $C_{i}: X_{i} \rightrightarrows U_{i}$ for components $\Sigma_{i}, i=1,2$, and a set of initial states $\mathcal{B} \subseteq X$, such that $X^{*}$ is uniformly attractive for system $\Sigma$ and controller $C$ from all initial states $x_{0} \in \mathcal{B}$.

In the following, we present a compositional approach to solve Problem 5. Throughout the paper, we assume that the transition systems $\Sigma_{i}$ are symbolic, i.e. $X_{i}$ and $U_{i}$ are finite sets for $i=1,2$. However, let us remark that our approach can also be used to synthesize controllers for systems with infinite sets of states and inputs by resorting to abstraction techniques (see e.g. [17], [13]), as shown in Section V.

\section{LEAST-VIOLATING ATTRACTIVITY CONTROLLERS}

In this section, we briefly recall results of [7] on the synthesis of attractivity controllers. While the results in [7] are stated for systems without external inputs, it is straightforward to extend them to this setting.

Consider a component $\Sigma_{i}=\left(X_{i}, D_{i}, U_{i}, F_{i}\right), i=1,2$, and a target set $X_{i}^{*}$. We assume that $X_{i}$ is equipped with a metric 
$d_{i}$, let us consider function $h_{i}: X_{i} \rightarrow \mathbb{R}_{\geq 0}$ such that $h_{i}\left(x_{i}\right)=0$ if and only if $x_{i} \in X_{i}^{*}$. An example of such function is

$$
h_{i}\left(x_{i}\right)=\min _{x_{i}^{\prime} \in X_{i}^{*}} d\left(x_{i}, x_{i}^{\prime}\right) .
$$

Let us consider the following sequence of dynamic programming fixed-point iterations:

$$
\begin{aligned}
W_{i, S}^{0}\left(x_{i}\right) & =h_{i}\left(x_{i}\right), \\
W_{i, S}^{k+1}\left(x_{i}\right) & = \begin{cases}\max \left(h_{i}\left(x_{i}\right), \min _{u_{i} \in \operatorname{enab}_{F_{i}}\left(x_{i}\right) x_{i}^{+} \in F_{i}\left(x_{i}, D_{i}, u_{i}\right)} W_{i, S}^{k}\left(x_{i}^{+}\right)\right) \\
& \text {if } \operatorname{enab}_{F_{i}}\left(x_{i}\right) \neq \emptyset ; \\
+\infty & \text { if } \operatorname{enab}_{F_{i}}\left(x_{i}\right)=\emptyset ;\end{cases}
\end{aligned}
$$

for $x_{i} \in X_{i}, k \in \mathbb{N}$. We denote the fixed-point of (1)-(2) by $W_{i, S}^{*}$. Then, let

$$
\begin{aligned}
W_{i, A}^{0}\left(x_{i}\right) & =W_{i, S}^{*}\left(x_{i}\right), \\
W_{i, A}^{k+1}\left(x_{i}\right) & = \begin{cases}\min \left(W_{i, S}^{*}\left(x_{i}\right), \min _{u_{i} \in \operatorname{enab}_{F_{i}}\left(x_{i}\right)} \max _{x_{i}^{+} \in F_{i}\left(x_{i}, D_{i}, u_{i}\right)} W_{i, A}^{k}\left(x_{i}^{+}\right)\right) \\
\text {if } \operatorname{enab}_{F_{i}}\left(x_{i}\right) \neq \emptyset ; \\
W_{i, S}^{*}\left(x_{i}\right) & \text { if } \operatorname{enab}_{F_{i}}\left(x_{i}\right)=\emptyset ;\end{cases}
\end{aligned}
$$

for $x_{i} \in X_{i}, k \in \mathbb{N}$. We denote the fixed-point of (3)-(4) by $W_{i, A}^{*}$.

It has been shown in [7] that for a symbolic system $\Sigma_{i}$, there exists $K_{i} \in \mathbb{N}$, such that for all $x_{i} \in X_{i}$, for all $k \geq K_{i}$, $W_{i, S}^{k}\left(x_{i}\right)=W_{i, S}^{*}\left(x_{i}\right)$ and $W_{i, A}^{k}\left(x_{i}\right)=W_{i, A}^{*}\left(x_{i}\right)$. Hence, $W_{i, S}^{*}$ and $W_{i, A}^{*}$ can be computed in practice. Then, let the function $k_{i}^{*}$ : $X_{i} \rightarrow \mathbb{N}$ be defined as follows for all $x_{i} \in X_{i}$

$$
k_{i}^{*}\left(x_{i}\right)=\min \left\{k \in \mathbb{N} \mid W_{i, A}^{k}\left(x_{i}\right)=W_{i, A}^{*}\left(x_{i}\right)\right\},
$$

and for $\delta \in \mathbb{R}$, let us consider the following controller for $\Sigma_{i}$

$$
C_{i}^{\delta}\left(x_{i}\right)=\left\{\begin{aligned}
& \min _{u_{i} \in \operatorname{enab}_{F_{i}}\left(x_{i}\right)}\left(\max _{x_{i}^{+} \in F_{i}\left(x_{i}, D_{i}, u_{i}\right)} W_{i, A}^{k_{i}^{*}\left(x_{i}\right)-1}\left(x_{i}^{+}\right)\right) \\
& \text {if } W_{i, A}^{*}\left(x_{i}\right) \leq \delta<W_{i, S}^{*}\left(x_{i}\right) ; \\
&\left\{u_{i} \in \operatorname{enab}_{F_{i}}\left(x_{i}\right) \max _{x_{i}^{+} \in F_{i}\left(x_{i}, D_{i}, u_{i}\right)} W_{i, S}^{*}\left(x_{i}^{+}\right) \leq \delta\right\} \\
& \text { if } W_{i, S}^{*}\left(x_{i}\right) \leq \delta ; \\
& \operatorname{enab}_{F_{i}}\left(x_{i}\right) \text { if } \delta<W_{i, A}^{*}\left(x_{i}\right) .
\end{aligned}\right.
$$

We state the following claim that will be useful in further discussions and that follows directly from (5) and (2):

Claim 6: For all $x_{i} \in X_{i}, C_{i}^{\delta}\left(x_{i}\right)=\emptyset$ if and only if $\operatorname{enab}_{F_{i}}\left(x_{i}\right)=\emptyset$.

We also recall the following result of [7]:
Theorem 7: There exists $T_{i} \in \mathbb{N}$, such that for all $x_{i, 0} \in$ $X_{i}$ with $W_{i, A}^{*}\left(x_{i, 0}\right) \leq \delta$, all maximal trajectories $\left(x_{i, t}\right)_{t=0}^{T} \in$ $\mathscr{T}_{\max }\left(\Sigma_{i}, C_{i}^{\delta}, x_{i, 0}\right)$ are complete and satisfy

$$
\forall t \geq T_{i}, W_{i, S}^{*}\left(x_{i, t}\right) \leq \delta .
$$

Hence, the set $\mathcal{A}_{i}=\left\{x_{i} \in X_{i} \mid W_{i, S}^{*}\left(x_{i}\right) \leq \delta\right\}$ is uniformly attractive for system $\Sigma_{i}$ and controller $C_{i}^{\delta}$ from all initial states $x_{i, 0} \in \mathcal{B}_{i}=\left\{x_{i} \in X_{i} \mid W_{i, A}^{*}\left(x_{i}\right) \leq \delta\right\}$. We refer to $\mathcal{A}_{i}$ as the attractor and to $\mathcal{B}_{i}$ as the basin of attraction.

It follows from $h_{i}\left(x_{i}\right) \leq W_{i, S}^{*}\left(x_{i}\right)$ that $\mathcal{A}_{i} \subseteq\left\{x_{i} \in X_{i} \mid h\left(x_{i}\right) \leq\right.$ $\delta\}$. Hence, if $\delta=0$, then $\mathcal{A}_{i} \subseteq X_{i}^{*}$ and $X_{i}^{*}$ is also uniformly attractive for system $\Sigma_{i}$ and controller $C_{i}^{\delta}$ from all initial states $x_{i, 0} \in \mathcal{B}_{i}$. It is shown in [7] that the controller $C_{i}^{\delta}$ is least-violating in the sense that $W_{i, A}^{*}\left(x_{i, 0}\right) \leq \delta$ if and only if there exists a controller for $\Sigma_{i}$ making the set $\left\{x_{i} \in\right.$ $\left.X_{i} \mid h\left(x_{i}\right) \leq \delta\right\}$ uniformly attractive from $x_{i, 0}$. Hence, the value of $W_{i, A}^{*}\left(x_{i, 0}\right)$ gives us the smallest neighborhood of $X_{i}^{*}$ which we can attract the trajectories of $\Sigma_{i}$ to.

We end the section by pointing out that the controller $C_{i}^{\delta}$ is set-valued and may provide more than one input, in particular for states belonging to the attractor.

\section{COMPOSITIONAL SYNTHESIS FOR ATTRACTIVITY}

In this section, we present an approach for synthesizing compositionally controllers solving Problem 5. The main idea of our approach is to compute iteratively refinements of least violating attractivity controllers defined in the previous section, resulting in a nested sequence of attractors eventually contained in the target set. Let us consider two components $\Sigma_{i}=\left(X_{i}, D_{i}, U_{i}, F_{i}\right), i=1,2$ with $D_{1}=X_{2}$ and $D_{2}=X_{1}$.

\section{A. Initialization of controllers}

For $i=1,2$, we start by computing $W_{i, S}^{*, 0}$ and $W_{i, A}^{*, 0}$, the fixed-point of equations (1)-(2) and (3)-(4), respectively, for system $\Sigma_{i}$. Then, let us define

$$
\begin{aligned}
\ell_{i}^{0} & =\min _{x_{i} \in X_{i}} W_{i, S}^{*, 0}\left(x_{i}\right) \\
\mathcal{A}_{i}^{0} & =\left\{x_{i} \in X_{i} \mid W_{i, S}^{*, 0}\left(x_{i}\right) \leq \ell_{i}^{0}\right\} \\
\mathcal{B}_{i} & =\left\{x_{i} \in X_{i} \mid W_{i, A}^{*, 0}\left(x_{i}\right) \leq \ell_{i}^{0}\right\}
\end{aligned}
$$

and let $C_{i}^{0}$ be the controller for $\Sigma_{i}$ defined as in (5) for $\delta=\ell_{i}^{0}$.

Let $\mathcal{A}^{0}=\mathcal{A}_{1}^{0} \times \mathcal{A}_{2}^{0}, \mathcal{B}=\mathcal{B}_{1} \times \mathcal{B}_{2}$ and let the decentralized controller for $\Sigma$ be given for all $x=\left(x_{1}, x_{2}\right) \in X$ by $C^{0}(x)=$ $C_{1}^{0}\left(x_{1}\right) \times C_{2}^{0}\left(x_{2}\right)$.

Proposition 8: There exists $T^{0} \in \mathbb{N}$, such that for all $x_{0} \in \mathcal{B}$, all maximal trajectories $\left(x_{t}\right)_{t=0}^{T} \in \mathscr{T}_{\max }\left(\Sigma, C^{0}, x_{0}\right)$ are complete and satisfy $x_{t} \in \mathcal{A}^{0}$, for all $t \geq T^{0}$.

Proof: Let $T_{i} \in \mathbb{N}$ be given as in Theorem 7 for $\delta=\ell_{i}^{0}, i=1,2$ and $T^{0}=\max \left(T_{1}, T_{2}\right)$. Let us consider $x_{0} \in \mathcal{B}$ 
and a maximal trajectory $\left(x_{t}\right)_{t=0}^{T} \in \mathscr{T}_{\max }\left(\Sigma, C^{0}, x_{0}\right)$ where $x_{t}=\left(x_{1, t}, x_{2, t}\right)$. We can verify that $\left(x_{i, t}\right)_{t=0}^{T}$ is a closedloop trajectory of $\Sigma_{i}$ with controller $C_{i}^{0}, i=1,2$. Moreover, maximality of $\left(x_{t}\right)_{t=0}^{T}$ implies that $\left(x_{1, t}\right)_{t=0}^{T}$ or $\left(x_{2, t}\right)_{t=0}^{T}$ is maximal. Let us assume without loss of generality that $\left(x_{1, t}\right)_{t=0}^{T}$ is maximal (the other case is symmetric). Since $W_{1, A}^{*, 0}\left(x_{1,0}\right) \leq \ell_{1}^{0}$, it follows from Theorem 7 that $\left(x_{1, t}\right)_{t=0}^{T}$ is complete and that $W_{1, S}^{*, 0}\left(x_{1, t}\right) \leq \ell_{1}^{0}$, for all $t \geq T_{1}$. Completeness of $\left(x_{1, t}\right)_{t=0}^{T}$ implies that $T=+\infty$ and therefore $\left(x_{2, t}\right)_{t=0}^{T}$ is also complete and thus maximal. Since $W_{2, A}^{*, 0}\left(x_{2,0}\right) \leq \ell_{2}^{0}$, it follows from Theorem 7 that $W_{2, S}^{*, 0}\left(x_{2, t}\right) \leq \ell_{2}^{0}$, for all $t \geq T_{2}$. Hence, $\left(x_{t}\right)_{t=0}^{T} \in \mathscr{T}_{\max }\left(\Sigma, C^{0}, x_{0}\right)$ is complete and $x_{t} \in \mathcal{A}^{0}$, for all $t \geq T^{0}$.

Let us remark that if $\mathcal{A}^{0} \subseteq X^{*}$, then the controllers $C_{i}^{0}$, $i=1,2$, solve Problem 5. If this is not the case, we can try to refine these least-violating attractivity controllers. Indeed, the controllers $C_{i}^{0}$ are set-valued and we can exploit this nondeterminism for further design to improve the closed-loop behavior of the system. In particular, knowing that after some time the states of the components are in the attractor $\mathcal{A}^{0}$, we can refine the controller $C_{i}^{0}$ by synthesizing a least violating attractivity controller for component $\Sigma_{i}$ when its external input belongs to the attractor of the other component. This procedure can then be repeated iteratively, as shown in the next section.

\section{$B$. Iterative refinement of controllers}

In the following, we use the following notational convention $1^{c}=2$ and $2^{c}=1$.

For $\alpha \in \mathbb{N}$, starting from $\alpha=0$, for $i=1,2$, let us define the systems $\Sigma_{i}^{\alpha+1}=\left(X_{i}, \mathcal{A}_{i^{c}}^{\alpha}, U_{i}, F_{i}^{\alpha+1}\right)$ where

$$
F_{i}^{\alpha+1}\left(x_{i}, d_{i}, u_{i}\right)= \begin{cases}F_{i}\left(x_{i}, d_{i}, u_{i}\right) & \text { if } u_{i} \in C_{i}^{\alpha}\left(x_{i}\right) \\ \emptyset & \text { if } u_{i} \notin C_{i}^{\alpha}\left(x_{i}\right)\end{cases}
$$

Intuitively, $\Sigma_{i}^{\alpha+1}$ describes the dynamics of component $\Sigma_{i}$ where external inputs are constrained by the attractor $\mathcal{A}_{i^{c}}^{\alpha}$ and the control inputs are constrained by the controller $C_{i}^{\alpha}$. We then compute least violating attractivity controllers for these systems. Let $W_{i, S}^{*, \alpha+1}$ and $W_{i, A}^{*, \alpha+1}$ be the fixed-point of equations (1)-(2) and (3)-(4), respectively, for system $\Sigma_{i}^{\alpha+1}$. Then, let us define

$$
\begin{aligned}
\ell_{i}^{\alpha+1} & =\max _{x_{i} \in \mathcal{A}_{i}^{\alpha}} W_{i, A}^{*, \alpha+1}\left(x_{i}\right) \\
\mathcal{A}_{i}^{\alpha+1} & =\left\{x_{i} \in \mathcal{A}_{i}^{\alpha} \mid W_{i, S}^{*, \alpha+1}\left(x_{i}\right) \leq \ell_{i}^{\alpha+1}\right\}
\end{aligned}
$$

and let $C_{i}^{\alpha+1}$ be the controller for $\Sigma_{i}^{\alpha+1}$ defined as in (5) for $\delta=\ell_{i}^{\alpha+1}$.
Claim 9: For $i=1,2, \alpha \in \mathbb{N}$, the following hold:

- $\mathcal{A}_{i}^{\alpha+1} \subseteq \mathcal{A}_{i}^{\alpha}$

- for all $x_{i} \in X_{i}, C_{i}^{\alpha+1}\left(x_{i}\right) \subseteq C_{i}^{\alpha}\left(x_{i}\right)$,

- for all $x_{i} \in X_{i}, C_{i}^{\alpha+1}\left(x_{i}\right)=\emptyset$ if and only if $C_{i}^{\alpha}\left(x_{i}\right)=\emptyset$.

Proof: The first item is a direct consequence of (11). From (9), we have that enab $F_{i}^{\alpha+1}\left(x_{i}\right)=C_{i}^{\alpha}\left(x_{i}\right)$. Then, the second item comes from (5) that gives $C_{i}^{\alpha+1}\left(x_{i}\right) \subseteq \operatorname{enab}_{F_{i}}^{\alpha+1}\left(x_{i}\right)$. The third item comes from Claim 6 that gives $C_{i}^{\alpha+1}\left(x_{i}\right)=\emptyset$ if and only if enab $F_{i}^{\alpha+1}\left(x_{i}\right)=\emptyset$.

Let $\mathcal{A}^{\alpha+1}=\mathcal{A}_{1}^{\alpha+1} \times \mathcal{A}_{2}^{\alpha+1}$, and let the decentralized controller for $\Sigma$ be given for all $x=\left(x_{1}, x_{2}\right) \in X$ by $C^{\alpha+1}(x)=$ $C_{1}^{\alpha+1}\left(x_{1}\right) \times C_{2}^{\alpha+1}\left(x_{2}\right)$. We can state the following result:

Theorem 10: For $\alpha \in \mathbb{N}$, there exists $T^{\alpha} \in \mathbb{N}$, such that for all $x_{0} \in \mathcal{B}$, all maximal trajectories $\left(x_{t}\right)_{t=0}^{T} \in \mathscr{T}_{\max }\left(\Sigma, C^{\alpha}, x_{0}\right)$ are complete and satisfy $x_{t} \in \mathcal{A}^{\alpha}$, for all $t \geq T^{\alpha}$.

Proof: We proceed by induction. The case $\alpha=0$ corresponds to Proposition 8. Then, let us assume that the property is true for some $\alpha \in \mathbb{N}$, that is that there exists $T^{\alpha} \in \mathbb{N}$, such that for all $x_{0} \in \mathcal{B}$, all maximal trajectories $\left(x_{t}\right)_{t=0}^{T} \in \mathscr{T}_{\max }\left(\Sigma, C^{\alpha}, x_{0}\right)$ are complete and satisfy $x_{t} \in \mathcal{A}^{\alpha}$, for all $t \geq T^{\alpha}$. From Claim 9, we have for all $x \in X$, $C^{\alpha+1}(x) \subseteq C^{\alpha}(x)$ and $C^{\alpha+1}(x)=\emptyset$ if and only if $C^{\alpha}(x)=\emptyset$, then it follows that for all $x_{0} \in \mathcal{B}$, all maximal trajectories $\left(x_{t}\right)_{t=0}^{T} \in \mathscr{T}_{\max }\left(\Sigma, C^{\alpha+1}, x_{0}\right)$ are complete and satisfy $x_{t} \in \mathcal{A}^{\alpha}$, for all $t \geq T^{\alpha}$. Let us denote $x_{t}=\left(x_{1, t}, x_{2, t}\right)$, then for all $t \geq T^{\alpha}, x_{i, t} \in \mathcal{A}_{i}^{\alpha}, i=1,2$. For $i=1,2$, we can then verify that $\left(x_{i, t}\right)_{t=T^{\alpha}}^{T}$ is closed-loop trajectory of $\Sigma_{i}^{\alpha+1}$ with controller $C_{i}^{\alpha+1}$. Moreover, $x_{i, T^{\alpha}} \in \mathcal{A}_{i}^{\alpha}$ implies by (10) that $W_{i, A}^{*, \alpha+1}\left(x_{i, T^{\alpha}}\right) \leq \ell_{i}^{\alpha+1}$, therefore it follows that $W_{i, S}^{*, \alpha+1}\left(x_{i, t}\right) \leq \ell_{i}^{\alpha+1}$, for all $t \geq T^{\alpha}+T_{i}$, where $T_{i}$ is the uniform time bound given in Theorem 7. Letting $T^{\alpha+1}=$ $T^{\alpha}+\max \left(T_{1}, T_{2}\right)$ we get from (11) that $x_{t} \in \mathcal{A}^{\alpha+1}$, for all $t \geq T^{\alpha+1}$.

Our approach thus computes successive refinements of least-violating controllers $C_{i}^{\alpha}$ associated to a sequence of nested attractors $\mathcal{A}^{\alpha}$. This allows us to build attractors that get closer to the target set $X^{*}$, and that may eventually be contained in $X^{*}$.

\section{Algorithm}

Our overall approach to synthesize decentralized attractivity controllers compositionally can be summarized in Algortihm 1.

We can now state the main result of the paper:

Theorem 11: Algorithm 1 terminates in a finite number of steps and the following properties hold: 


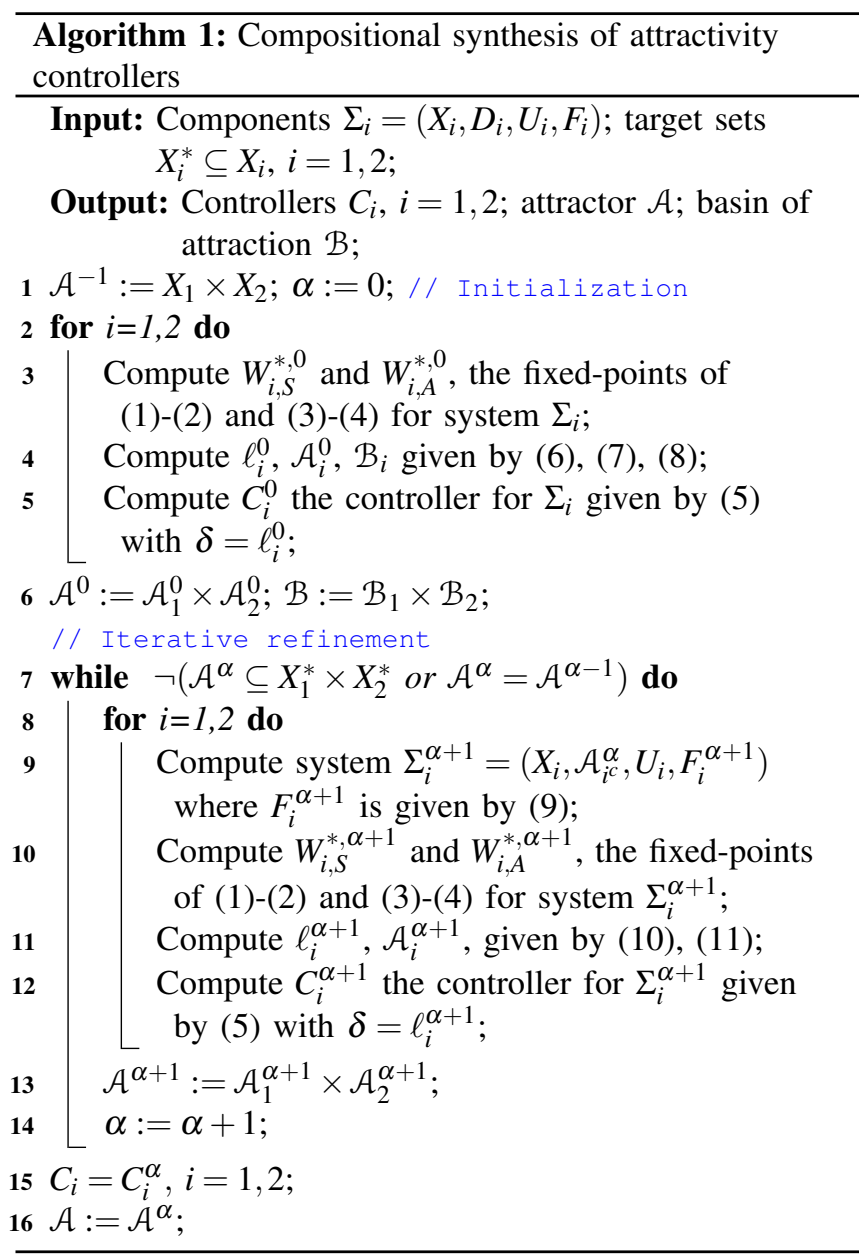

- $\mathcal{A}$ is uniformly attractive for system $\Sigma$ and controller $C$ from all initial states $x_{0} \in \mathcal{B}$.

- If $\mathcal{A} \subseteq X^{*}, C_{1}$ and $C_{2}$ are solutions to Problem 5 .

Proof: From Claim 9, we get that $\mathcal{A}^{\alpha} \subseteq \mathcal{A}^{\alpha-1}$ for all $\alpha \geq 0$. Then since the transition systems are symbolic, $X_{1} \times X_{2}$ is a finite set. Hence, there exists $\alpha \geq 0$ such that $\mathcal{A}^{\alpha}=\mathcal{A}^{\alpha-1}$. It follows that the while loop terminates after a finite number of iterations. Hence, Algorithm 1 terminates in a finite number of steps. Then, the first item is a direct consequence of Theorem 10 and the second item follows from the first item.

\section{NUMERICAL EXAMPLE}

In this section, we show an illustrative application of Algorithm 1 to a model of temperature regulation in a four room building. We model the building as an interconnected systems of two components, each component consisting of two rooms. The continuous-time dynamics is given by:

$$
\begin{aligned}
& \dot{x}_{1}=\alpha A\left(x_{1}+x_{2}\right)-2 \alpha x_{1}+\beta\left(T_{e} \mathbf{1}-x_{1}\right)+\Gamma\left(u_{1}\right)\left(T_{h_{1}}-x_{1}\right) \\
& \dot{x}_{2}=\alpha A\left(x_{1}+x_{2}\right)-2 \alpha x_{2}+\beta\left(T_{e} \mathbf{1}-x_{2}\right)+\Gamma\left(u_{2}\right)\left(T_{h_{2}}-x_{2}\right)
\end{aligned}
$$

where $x_{1}=\left[\begin{array}{ll}T_{1} & T_{2}\end{array}\right]^{T}$ and $x_{2}=\left[T_{3} T_{4}\right]^{T}$ represent the temperatures in the rooms $1,2,3$ and $4 ; T_{e}$ is the temperature of the external environment of the building; $T_{h_{1}}=\left[\tilde{T}_{h_{1}} \tilde{T}_{h_{2}}\right]^{T}$ and $T_{h_{2}}=\left[\begin{array}{ll}\tilde{T}_{h_{3}} & \tilde{T}_{h_{4}}\end{array}\right]^{T}$ represent the maximal temperature of the

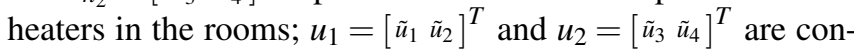
trol inputs representing the heating power used in the rooms with 0 corresponding to no heating and 1 corresponding to full power. $A=\left[\begin{array}{ll}0 & 1 \\ 1 & 0\end{array}\right]$ represents the interconnections between rooms, $\mathbf{1}=\left[\begin{array}{ll}1 & 1\end{array}\right]^{T}, \Gamma(v)=\left[\begin{array}{cc}\gamma \tilde{v}_{1} & 0 \\ 0 & \gamma \tilde{v}_{2}\end{array}\right]$ where $v=\left[\begin{array}{cc}\tilde{v}_{1} & \tilde{v}_{2}\end{array}\right]^{T} . \alpha, \beta$ and $\gamma$ are the conduction factors between the rooms, between the external environment and the rooms and between the heaters and the rooms, respectively. The values of $\alpha, \beta, \gamma$ are respectively $1 / 20,1 / 200,1 / 100 ; T_{e}=10^{\circ} \mathrm{C}, \tilde{T}_{h_{1}}=50^{\circ} \mathrm{C}$, $\tilde{T}_{h_{2}}=80^{\circ} \mathrm{C}, \tilde{T}_{h_{3}}=70^{\circ} \mathrm{C}, \tilde{T}_{h_{4}}=100^{\circ} \mathrm{C}$.

The continuous-time dynamics of (12) is periodically sampled with period 5 minutes. We impose the state constraint $T_{i} \in[16,24], i=1, \ldots, 4$ and the control objective is to stabilize the temperature of all rooms in the intervals $[19,21]$. We compute symbolic abstractions of both components using the approach described in [11]. For that purpose, we use uniform partitions of the state intervals $[16,24]$ in $100 \mathrm{sub}$ intervals and uniform discretizations of the input intervals $[0,1]$ with 5 elements. Each component has then $10^{4}$ states and 25 inputs.

We use Algorithm 1 to synthesize an attractivity controller for the system. The algorithm stops after 6 iterations of the main loop after the computed attractor is contained in the target set $[19,21]^{4}$. The total computation time is 45 seconds, with 13 seconds spent on computing abstractions of components, 11 seconds spent on initializing the controller and 22 seconds spent in refining the controller iteratively. We show on Figure 1 the sets that have been computed, where the black region represents uncontrollable states (i.e. states without guarantees of ever reaching the attractors), the blue region is the basin of attraction and the other regions are the sequence of attractors with the final attractor represented in yellow and contained in the target set in red.

Assuming initial temperatures $x_{1}(0)=\left[\begin{array}{ll}16 & 23\end{array}\right]^{T}$ and $x_{2}(0)=\left[\begin{array}{lll}16.5 & 22.5\end{array}\right]^{T}$ for the rooms, Figure 1 shows the trajectories of the room's temperatures in the state space with respect to the time, while applying the attractivity controllers obtained from Algorithm 1. 

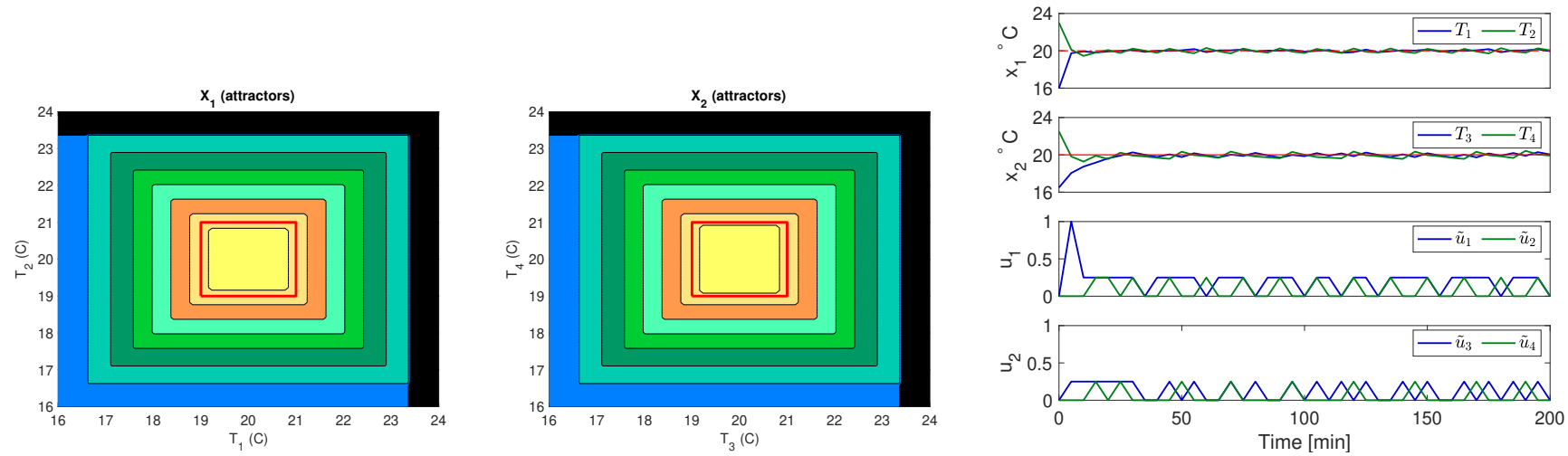

Fig. 1. (Left and middle) Sets computed by Algorithm 1, black region represents uncontrollable states (i.e. states without guarantees of ever reaching the attractors), the blue region is the basin of attraction and the other regions are the sequence of attractors with the final attractor represented in yellow and contained in the target set in red. (Right) Simulations (state and input evolutions) of the closed-loop system with initial condition $x_{1}(0)=[1623]^{T}$ and $x_{2}(0)=\left[\begin{array}{lll}16.5 & 22.5\end{array}\right]^{T}$.

\section{CONCLUSIONS}

In this work, we presented a novel compositional approach to the synthesis of decentralized attractivity controllers. While the paper focuses on systems with two components, the approach can easily be extended to an arbitrary number of components. The approach is based on iterative refinements of least-violating attractivity controllers and of the associated attractors. We presented an algorithm for systems with finite state and input spaces and we have shown, using an example, how our approach can also be used to design controllers for systems with infinite state and input spaces using symbolic abstractions. Future work will focus on extending the approach to other types of specifications and to other types of interconnected systems such as systems with overlapping components as in [11], [14].

\section{REFERENCES}

[1] W. A. Apaza-Perez, C. Combastel, and A. Zolghadri. Abstractionbased low complexity controller synthesis for interconnected nondeterministic systems. In European Control Conference, pages 4174 4179, 2019.

[2] W. A. Apaza-Perez, C. Combastel, and A. Zolghadri. On distributed symbolic control of interconnected systems under persistency specifications. International Journal of Applied Mathematics and Computer Science, 30(4), 2020.

[3] C. Belta, B. Yordanov, and E. Gol. Formal Methods for Discrete-Time Dynamical Systems. Springer, 2017.

[4] A. Benveniste, B. Caillaud, D. Nickovic, R. Passerone, J.-B. Raclet, P. Reinkemeier, A. Sangiovanni-Vincentelli, W. Damm, T. A. Henzinger, and K. G. Larsen. Contracts for system design. Foundations and Trends in Electronic Design Automation, 12(2-3):124-400, 2018.

[5] E. Dallal and P. Tabuada. On compositional symbolic controller synthesis inspired by small-gain theorems. In IEEE Conference on Decision and Control, pages 6133-6138, 2015.
[6] A. Eqtami and A. Girard. A quantitative approach on assumeguarantee contracts for safety of interconnected systems. In European Control Conference, pages 536-541. IEEE, 2019.

[7] A. Girard and A. Eqtami. Least-violating symbolic controller synthesis for safety, reachability and attractivity specifications. Automatica, 127:109543, 2021.

[8] E. S. Kim, S. Sadraddini, C. Belta, M. Arcak, and S. A. Seshia Dynamic contracts for distributed temporal logic control of traffic networks. In IEEE Conference on Decision and Control, pages 36403645, 2017.

[9] K. Mallik, A.-K. Schmuck, S. Soudjani, and R. Majumdar. Compositional synthesis of finite-state abstractions. IEEE Transactions on Automatic Control, 64(6):2629-2636, 2018.

[10] P.-J. Meyer and D. V. Dimarogonas. Compositional abstraction refinement for control synthesis. Nonlinear Analysis: Hybrid Systems, 27:437-451, 2018.

[11] P. J. Meyer, A. Girard, and E. Witrant. Compositional abstraction and safety synthesis using overlapping symbolic models. IEEE Transactions on Automatic Control, 63(6):1835-1841, 2018.

[12] G. Pola, P. Pepe, and M. D. Di Benedetto. Symbolic models for networks of control systems. IEEE Transactions on Automatic Control, 61(11):3663-3668, 2016.

[13] G. Reissig, A. Weber, and M. Rungger. Feedback refinement relations for the synthesis of symbolic controllers. IEEE Transactions on Automatic Control, 62(4):1781-1796, 2017.

[14] A. Saoud, A. Girard, and L. Fribourg. Contract-based design of symbolic controllers for safety in distributed multiperiodic sampleddata systems. IEEE Transactions on Automatic Control, 66(3):10551070, 2021.

[15] A. Saoud, P. Jagtap, M. Zamani, and A. Girard. Compositional abstraction-based synthesis for interconnected systems: An approximate composition approach. IEEE Transactions on Control of Network Systems, 2021.

[16] A. Swikir and M. Zamani. Compositional synthesis of finite abstractions for networks of systems: A small-gain approach. Automatica, 107:551-561, 2019.

[17] P. Tabuada. Verification and Control of Hybrid Systems. Springer, 2009

[18] Y. Tazaki and J.-i. Imura. Bisimilar finite abstractions of interconnected systems. In International Workshop on Hybrid Systems: Computation and Control, pages 514-527. Springer, 2008. 